

\title{
A LEAST-SQUARES FINITE ELEMENT METHOD FOR INCOMPRESSIBLE NAVIER-STOKES PROBLEMS
}

\author{
Bo-nan Jiang* \\ Institute for Computational Mechanics in Propulsion \\ Lewis Research Center \\ Cleveland, Ohio $\mathbf{4 4 1 3 5}$
}

\section{SUMMARY}

A least-squares finite element method, based on the velocity-pressure-vorticity formulation, is developed for solving steady incompressible Navier-Stokes problems. This method leads to a minimization problem rather than to a saddle-point problem by the classic mixed method, and can thus accommodate equal-order interpolations. This method has no parameter to tune. The associated algebraic system is symmetric, and positive definite. Numerical results for the cavity flow at Reynolds number up to 10,000 and the backward-facing step flow at Reynolds number up to 900 are presented.

\section{INTRODUCTION}

During the past decades various finite element methods for incompressible viscous flows have been developed. Extensive results can be found in the literature. ${ }^{1-6}$ Most of these finite element methods are based on the velocity-pressure formulation because of its simpler boundary conditions and easier extension to three-dimensions. Three methods are commonly used to solve the velocity-pressure equations. They are the Galerkin mixed method, the penalty method and the segregated method.

In the Galerkin mixed method, different elements have to be used to interpolate the velocity and the pressure in order to satisfy the Ladyzhenskaya-Babuška-Brezzi(LBB) condition for the existence of the solution. ${ }^{7,8}$ Although for two-dimensional problems quite a few convergent pairs of velocity and pressure elements have been developed, most of these combinations employ basis functions that are not convenient to implement. For three dimensional problems, this difficulty becomes more severe and only rather elaborate constructions can pass the LBB test. Another difficulty is that the matrix associated with the system of linear equations is both non-symmetric due to the convection terms in the Navier-Stokes equations and non-positive-definite owing to the uncoupled nature of the incompressibility constraint. Therefore, direct Gaussian elimination rather than iterative techniques has been considered the only viable method for solving the system. But, for three-dimensional problems, the computer resources required by a direct method become prohibitively large.

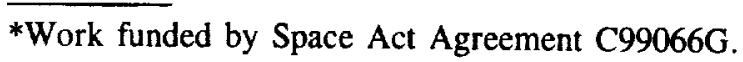


In the penalty method, the pressure is pre-eliminated by penalizing the continuity equation. Involving only velocities, a considerable saving in computing time and computer memory is achieved. However, in many engineering applications, the pressure may be the most important design parameter, but the pressure recovered by using the perturbed conservation of mass equation exhibits oscillations due to the ill-conditioned pressure matrix. Another disadvantage is the penalty parameter, which for small values causes loss of accuracy and for too large values sometimes prevents convergence to the solution. Furthermore, because of the ill-condition due to the smallness of the parameter, the linear system can not be solved by iterative techniques. Thus one can not use the penalty method to solve large-scale problems.

The segregated method ${ }^{9,10,11}$ adopts a well-known SIMPLE-type finite difference algorithm. Since the computation of velocity and pressure are decoupled by iteration, signifcant computational efficiency can be achieved in computer storage and time. However, due to the lack of correct pressure boundary conditions for the pressure correction equation, the computed pressure may be inaccurate. A comparative study of the segregated method vs the Galerkin mixed method can be found in Reference 12 .

In order to overcome these difficulties, we have been developing a least-squares finite element method(LSFEM) ${ }^{13,14,15}$ based on the first-order velocity-pressure-vorticity formulation. Any good solver of Navier-Stokes equations should at least be able to solve the Stokes equations. For the Stokes equations, this method leads to a minimization problem rather than to a saddle-point problem, and the choice of combination of elements is thus not subject to the LBB condition. The numerical experiments exhibit the optimal rate of convergence for all variables with equal-order interpolations. A theoretical error analysis supports this observation. ${ }^{16}$ Furthermore, there are neither added parameters nor artificial dissipation nor upwinding. The resulting matrix is symmetric and positive definite. Therefore, simple iterative techniques can be employed to solve large-scale problems on vector and parallel computers.

In this paper, we describe the LSFEM which has all the above mentioned advantages for solving the incompressible Navier-Stokes problems. The numerical procedure used in the present study is based on a general framework of the LSFEM for systems of firstorder partial differential equations. ${ }^{17}$ For the sake of completeness, we disscus the LSFEM for first-order systems in Section 2. Section 3 provides a derivation of the first-order velocity-pressure-vorticity formulation for the Navier-Stokes equations. In Section 4 the performance of the LSFEM is illustrated by the computational results for high Reynolds 
number cavity flows and backward-facing step flows. Conclusions are given in Section 5 .

\section{LSFEM FOR FIRST-ORDER SYSTEMS}

The LSFEM of interest here is based on minimizing the residual in differential equations in $L^{2}$ norm. The LSFEM requires that the trial functions be smooth enough to lie in the domain of the differential operator. For example, for second-order differential equations, the LSFEM must use $C^{1}$ elements(plate or shell elements) which are not convenient from the computational point of view. In order to use simple $C^{0}$ elements, we transform the original differential equations into an equivalent system of first-order differential equations by introducing auxiliary unknowns. This can be realized for almost any governing equation in mathematical physics.

We consider the boundary-value problem:

$$
\begin{array}{ll}
\mathbf{L} \underline{u}=\underline{f} & \text { in } \Omega \\
\mathbf{B} \underline{u}=\underline{g} & \text { on } \Gamma
\end{array}
$$

where $\mathbf{L}$ is a first-order partial differential operator:

$$
\mathbf{L} \underline{u}=\sum_{i=1}^{n_{d}} \mathbf{A}_{i} \frac{\partial \underline{u}}{\partial x_{i}}+\mathbf{A} \underline{u}
$$

$\Omega \subset \Re^{n_{d}}$ is a bounded domain with a piecewise smooth boundary $\Gamma, n_{d}=2$ or 3 represents the number of space dimensions, $\underline{u}^{T}=\left(u_{1}, u_{2}, \ldots u_{m}\right)$ is a vector of $m$ unknown functions of $\underline{x}, \mathbf{A}_{i}$ and $\mathbf{A}$ are $m \times m$ matrices which depend on $\underline{x}, f$ is a given vector-valued function, $\mathbf{B}$ is a boundary operator, and $\underline{g}$ is a given vector-valued function on the boundary. Without loss of generality we assume that $\underline{g}$ is a zero vector.

Here, we do not discuss the existence and uniqueness of the solution to (1), because these depend on the structure and properties of $\mathbf{L}$ and $\mathbf{B}$, and the vector $f$. In the following discussion, it is assumed that the problem (1) has a unique solution. We indicate that if there is a solution to (1), then the following least-squares method produces an approximate solution.

Throughout this paper, $L^{2}(\Omega)$ denotes the space of square-integrable functions defined on $\Omega$ with inner product

$$
(u, v)=\int_{\Omega} u v d \Omega \quad u, v \in L^{2}(\Omega)
$$


and norm

$$
\|u\|_{0}^{2}=(u, u) \quad u \in L^{2}(\Omega)
$$

We define the Sobolev space as:

$$
H^{1}(\Omega)=\left\{u \in L^{2}(\Omega) ; \partial^{\alpha} u \in L^{2}(\Omega), \forall|\alpha| \leq 1\right\}
$$

where $\alpha=\left(\alpha_{1}, \alpha_{2}, \ldots, \alpha_{n_{d}}\right) \in \mathcal{N}^{n_{d}}$ and $|\alpha|=\alpha_{1}+\alpha_{2}+\ldots+\alpha_{n_{d}}$, and define their associated norms by

$$
\|u\|_{1}^{2}=\sum_{|\alpha| \leq 1}\left\|\partial^{\alpha} u\right\|_{0}^{2}
$$

For the vector-valued function $\underline{u}$ with $m$ components, we have the product spaces

$$
\begin{aligned}
\underline{L}^{2}(\Omega) & =\left(L^{2}(\Omega)\right)^{m} \\
\underline{H}^{1}(\Omega) & =\left(H^{1}(\Omega)\right)^{m}
\end{aligned}
$$

and the corresponding norm

$$
\begin{aligned}
& \|\underline{u}\|_{0}^{2}=\sum_{j=1}^{m}\left\|u_{j}\right\|_{0}^{2} \\
& \|\underline{u}\|_{1}^{2}=\sum_{j=1}^{m}\left\|u_{j}\right\|_{1}^{2}
\end{aligned}
$$

Considering the boundary condition of the boundary-value problem, we also define the function space

$$
\underline{S}=\left\{\underline{u} \in\left(H^{1}(\Omega)\right)^{m} ; \mathbf{B} \underline{u}=0 \text { on } \Gamma\right\}
$$

Let us suppose that $f \in \underline{L}^{2}$ and $\mathbf{L}: \underline{S} \rightarrow \underline{L}^{2}$. For an arbitrary trial function $\underline{u} \in \underline{S}$, we define the residual function $\underline{R}=\mathbf{L} \underline{u}-\underline{f}$. The LSFEM is based on minimizing the residual function in a least-squares sense.

We construct the least-squares functional

$$
I(\underline{u})=\|\mathbf{L} \underline{u}-\underline{f}\|_{0}^{2}=(\mathbf{L} \underline{u}-\underline{f}, \mathbf{L} \underline{u}-\underline{f})
$$

Taking variation of $I$ with respect to $\underline{u}$, and setting $\delta I=0$ and $\delta \underline{u}=\underline{w}$, lead to the least-squares weak statement: Find $\underline{u} \in \underline{S}$ such that

$$
(\mathbf{L} \underline{w}, \mathbf{L} \underline{u})=(\mathbf{L} \underline{w}, \underline{f}) \quad \forall \underline{w} \in \underline{S}
$$


In the approximate analysis, we first discretize the domain as a union of finite elements and then introduce an appropriate finite element basis. Let $N_{e}$ denote the number of nodes for one element and $\psi_{j}$ denote the element shape functions. If equal-order interpolations are employed, that is, for all unknown variables the same finite element is used, we can write the expansion

$$
\underline{u}_{h}(\underline{x})=\sum_{j=1}^{N_{e}} \psi_{j}(\underline{x})\left(\begin{array}{c}
u_{1} \\
u_{2} \\
\vdots \\
u_{m}
\end{array}\right)_{j}
$$

where $\left(u_{1}, u_{2}, \ldots, u_{m}\right)_{j}$ are the nodal values at the $\mathrm{jth}$ node, and $h$ denotes the mesh parameter.

Introducing the finite element approximation defined in (15) into the weak statement (14), we have the linear algebraic equations

$$
\mathbf{K U}=\mathbf{F}
$$

where the $\mathbf{U}$ is the global vector of nodal values. The global matrix $\mathbf{K}$ is assembled from the element matrices

$$
\mathbf{K}_{e}=\int_{\Omega_{e}}\left(\mathbf{L} \psi_{1}, \mathbf{L} \psi_{2}, \ldots, \mathbf{L} \psi_{N e}\right)^{T}\left(\mathbf{L} \psi_{1}, \mathbf{L} \psi_{2}, \ldots, \mathbf{L} \psi_{N e}\right) d \Omega
$$

in which $\Omega_{e} \subset \Omega$ is the domain of the eth element, $T$ denotes the transpose, and the vector $\mathbf{F}$ is assembled from the element vectors

$$
\mathbf{F}_{e}=\int_{\Omega_{e}}\left(\mathbf{L} \psi_{1}, \mathbf{L} \psi_{2}, \ldots, \mathbf{L} \psi_{N_{e}}\right)^{T} \underline{f} d \mathbf{\Omega}
$$

in which

$$
\mathbf{L} \psi_{j}=\psi_{j, x} \mathbf{A}_{1}+\psi_{j, y} \mathbf{A}_{2}+\psi_{j, z} \mathbf{A}_{3}+\psi_{j} \mathbf{A}
$$

REMARK 1 If $\mathbf{L}$ is an elliptic operator, then we have the inequality

$$
(\mathbf{L} \underline{w}, \mathbf{L} \underline{u}) \leq M_{0}\|\underline{w}\|_{1}\|\underline{u}\|_{1}, \quad(\mathbf{L} \underline{u}, \mathbf{L} \underline{u}) \geq \alpha\|\underline{u}\|_{1}^{2}
$$

for arbitrary $\underline{u}, \underline{w} \in S$, where $M_{0}$ and $\alpha$ are positive constants. That is, the bilinear form $(\mathbf{L} \underline{w}, \mathbf{L} \underline{u})$ is continuous and coercive. Following the classic argument(see,e.g., Reference 18, p.26-28), if the exact solution is smooth enough, we can easily obtain the error estimate

$$
\|\underline{e}\|_{1}=\left\|\underline{u}-\underline{u}_{h}\right\|_{1} \leq \frac{M_{0} C}{\alpha} h^{k}
$$


where the constant $C$ is independent of the mesh size $h$, and $k$ denotes the order of complete polynomial of shape functions. Furthermore, the $L^{2}$ error estimate can be obtained by the Aubin-Nitsche trick(see, e.g., Reference 19, p.88). These facts indicate that all variables with equal-order interpolations converge at the optimal rate.

REMARK 2. The matrix $K$ is symmetric and positive definite. Therefore, iterative techniques, such as the preconditioned element-by-element conjugate gradient method, ${ }^{20,21}$ can be employed to solve the resulting algebraic equations. This property means that largescale problems can be effectively solved on vector and parallel computers.

REMARK 3. The computational labor of iterative methods depends on the condition number of the matrix $\mathbf{K}$. If a second-order elliptic problem is solved numerically using the classic Galerkin method on a uniform finite element mesh of size $h$, the resulting matrix has condition number $O\left(h^{-2}\right)$. If the same problem is recast as a first-order system, and then treated by the LSFEM, the condition number of matrix $\mathrm{K}$ remains as $O\left(h^{-2}\right) \cdot{ }^{22,23}$ That is, the LSFEM described here does not degrade the condition number.

REMARK 4. It is important to point out that there is no weighting parameter in our LSFEM. Each equation in the first-order system is equally treated. That is, the LSFEM is robust (with no parameter to tune ). People thought that in least-squares methods one could put a large weighting factor on one equation in the system to emphasize the importance of this equation over others. ${ }^{24}$ Numerical experiments and theoretical analysis show that this idea is incorrect. The weighting can not change the minimum point(zero) of the least-squares functional, and thus can not change the solution except the condition number of the resulting matrix. ${ }^{23}$ Usually, the governing equations are nondimensionalized by using the reference quantities of the problem. The nondimensionalization or scaling is equivalent to weighting the residuals in the least-squares functional. By the same argument, the scaling with different choice of the reference quantities will not change the LSFEM solution.

REMARK 5 The formulation of LSFEM is simple and systematic. Once a LSFEM based on a first-order system is coded, adaptation of the program to other problems is simply carried out by modifying the subroutines associated with the coefficient matrices $\mathbf{A}_{1}, \mathbf{A}_{2}$, $\mathbf{A}_{3}$ and $\mathbf{A}$ and the vector function $\underline{f}$. In this way, one may develop a general-purpose program.

\section{VELOCITY-PRESSURE-VORTICITY FORMULATION}

Consider the incompressible Navier-Stokes problem: Find the velocity $\underline{u}=\left(u_{1}, u_{2}, u_{3}\right)$ and the pressure $p$ such that

$$
\nabla \cdot \underline{u}=0 \quad \text { in } \Omega
$$




$$
\underline{u} \cdot \nabla \underline{u}-\frac{1}{R e} \Delta \underline{u}+\nabla p=\underline{f} \quad \text { in } \Omega
$$

Here all variables are nondimensionalized, $\underline{f}=\left(f_{x}, f_{y}\right)$ is the body force, and $R e$ denotes the Reynolds number, defined as

$$
R e=\frac{U L}{\nu}
$$

where $L$ a reference length, $U$ a reference velocity and $\nu$ the kinematic viscosity. Of course, the boundary conditions should be supplemented to complete the definition of the boundary value problem.

Since the momentum equations(22b) involve second-order derivatives of velocity, the direct application of least-squares method requires the use of inconvenient $C^{1}$ elements and produces matrices with large condition number. ${ }^{22}$ In order to use the LSFEM described in Section 2, we must consider the governing equations of incompressible flows in the form of first-order systems. There are several ways to do this. For example, one may write the Navier-Stokes equations in velocity-pressure-stress formulation, which is useful for viscoelasticity and non-Newtonian flow problems, but this formulation has too many variables. Instead, we introduce the vorticity $\underline{\omega}=\nabla \times \underline{u}$, and rewrite the incompressible Navier-Stokes equations in the following first-order quasi-linear velocity-pressure-vorticity formulation:

$$
\begin{gathered}
\nabla \cdot \underline{u}=0 \\
\underline{u} \cdot \nabla \underline{u}+\frac{1}{R e} \nabla \times \underline{\omega}+\nabla p=\underline{f} \quad \text { in } \Omega \\
\underline{\omega}-\nabla \times \underline{u}=0
\end{gathered}
$$

We shall consider the two-dimensional problem only:

$$
\begin{gathered}
\frac{\partial u}{\partial x}+\frac{\partial v}{\partial y}=0 \\
u \frac{\partial u}{\partial x}+v \frac{\partial u}{\partial y}+\frac{\partial p}{\partial x}+\frac{1}{R e} \frac{\partial \omega}{\partial y}=f_{x} \\
u \frac{\partial v}{\partial x}+v \frac{\partial v}{\partial y}+\frac{\partial p}{\partial y}-\frac{1}{R e} \frac{\partial \omega}{\partial x}=f_{y} \quad \text { in } \Omega \\
\omega+\frac{\partial u}{\partial y}-\frac{\partial v}{\partial x}=0
\end{gathered}
$$

We can write(24) in the general form of a first-order system(1), in which

$$
\mathbf{A}_{1}=\left(\begin{array}{cccc}
1 & 0 & 0 & 0 \\
u & 0 & 1 & 0 \\
0 & u & 0 & -\frac{1}{R_{e}} \\
0 & -1 & 0 & 0
\end{array}\right) \quad \mathbf{A}_{2}=\left(\begin{array}{cccc}
0 & 1 & 0 & 0 \\
v & 0 & 0 & \frac{1}{R_{e}} \\
0 & v & 1 & 0 \\
1 & 0 & 0 & 0
\end{array}\right)
$$




$$
\mathbf{A}=\left(\begin{array}{llll}
0 & 0 & 0 & 0 \\
0 & 0 & 0 & 0 \\
0 & 0 & 0 & 0 \\
0 & 0 & 0 & 1
\end{array}\right) \quad \underline{\mathbf{f}}=\left(\begin{array}{c}
0 \\
f_{x} \\
f_{y} \\
0
\end{array}\right) \quad \underline{\mathbf{u}}=\left(\begin{array}{c}
u \\
v \\
p \\
\omega
\end{array}\right)
$$

Since the system is of first-order, the boundary conditions are thus very simple, and do not involve the derivatives of unknowns. Let $\left(\Gamma_{1}, \Gamma_{2}, \Gamma_{3}, \Gamma_{4}, \Gamma_{5}\right)$ denote the sides of $\Gamma$. The unit outward normal vector to $\Gamma$ is denoted by $\underline{n}$, and the tangential vector to $\Gamma$ by $\underline{t}$. We may consider, for instance, the following boundary conditions:

(a) $u=0, v=0$ on the $\Gamma_{1}$ (the wall)

(b) $u=$ constant, $v=0, \omega=0$ on $\Gamma_{2}$ (the far field)

(c) $u=$ given, $v=0, \omega=$ given on $\Gamma_{3}$ (the well developed inflow or outflow)

(d) $u_{n}=0, w=0, p=$ constant on $\Gamma_{4}$ (the free surface)

(e) $u_{t}=0, p=$ constant on $\Gamma_{5}$ (the outflow)

The quasi-linear problem(25) can be solved by using any linearization method, for example, the successive substitution or the Newton's scheme.

\section{NUMERICAL RESULTS}

The LSFEM described in the previous sections has been tested by solving the driven cavity flow and the backward-facing step flow. In this study, a simple successive substitution scheme is used to obtain the solution. The velocity field at the previous step is used to calculate the coefficient matrices $\mathbf{A}_{1}, \mathbf{A}_{2}$ and $\mathbf{A}$. This paper is concerned with the validity of the LSFEM only, thus a Gaussian elimination is used to solve the algebraic system. The solutions are updated using an under-relaxation method given as

$$
\underline{u}^{*}=\alpha \underline{u}^{n}+(1-\alpha) \underline{u}^{n-1}
$$

where $\alpha$ is the under-relaxation number, the superscript $n$ denotes the substitution level, and ${ }^{*}$ the updated solution. The difference between the results of two consecutive steps is defined as

$$
e=\max _{i=1, \cdots, N_{n}}\left|u_{i}^{n}-u_{i}^{n+1}\right|
$$

where $i$ denotes the node, $N_{n}$ is the total number of nodes. The substitution continues until the difference $e$ becomes less than the tolerance $10^{-4}$.

\section{Driven Cavity Flow}

The definition of driven cavity flow is as usual. $50 \times 50$ nonuniform bilinear elements are used and the mesh distribution is shown in Figure 1. The smallest element size has 
$h=0.002$ at the four coners. One point Gaussian quadrature is used to evaluate the element matrices. The boundary conditions are: $u=v=0$ everywhere except on the toplid, where $u=1, v=0 ; p=0$ at the center of the bottom; $\omega=0$ at the two lower corners; $\omega=-500$ at the two upper corners. The Reynolds numbers considered are $100,400,1000,3200,5000,7500$, and 10000 . In each case, $u=v=0$ is taken as an initial guess, that is, the first step is the solution of the corresponding Stokes problem. No underrelaxation is necessary for $R e<3200, \alpha=0.8$ is used for $R e \geq 3200$. The required number of iterations are $8,12,14,22,28,40$, and 67 , respectively.

The computed results(streamlines, pressure contours, vorticity contours, and velocity vectors) for $R e=1000,5000$ and 10000 are shown in Figure 2,3, and 4. Overall, the streamlines and the vorticity contours compare rather well with those of Ghia et al ${ }^{25}$ except for one region: the lower right eddy at $R e=10000$. The size and shape of this small eddy compare more favorably with those of Gresho et al. ${ }^{26}$ The pressure contours compare well with those of $\mathrm{Kim}^{27}$ and Sohn et al. ${ }^{12}$ The horizontal velocity profiles at $x=0.5$ compare well with those of Ghia et $a^{25}$ in Figure 5 .

\section{Backward-facing Step}

This example is chosen to compare computational results with the experimental data of Almaly et $a .^{28}$ The step has a height of $0.0049 \mathrm{~m}$. The small channel, upstream of this step, has a height of $0.0052 \mathrm{~m}$. the inlet boundary is located at 3.5 step heights upstream of this step. The total length behind the backward-facing step is 45 step heights. A total of 2550 nonuniform bilinear elements $(6 \times 15$ for the smaller channel and $82 \times 30$ for the larger channel) are used with fine meshes near the step. One point Gaussian quadrature is used to evaluate the element matrices.

A parabolic velocity profile with the center line velocity of $1.0 \mathrm{~m} / \mathrm{s}$ and a corresponding vorticity are imposed at the inlet. $v=0, p=0$ are prescribed at the exit boundary. $\omega=0$ is given at the lower step corner. The Reynolds number $R e=\frac{U D}{\nu}$ is based on the hydraulic $\operatorname{diameter}(D=0.0104 \mathrm{~m})$ of the inlet channel and the average velocity $(v=0.6667 \mathrm{~m} / \mathrm{s})$. The various Reynolds numbers are obtained by varying the kinematic viscosity $\nu$.

$u=v=0$ is used as an initial guess for $R e=100$, the converged solution for $R e=100$ is used as an initial guess for $R e=200$, and so on. The required numbers of substitutions are $13,19,29,39,42,51,73,79$, and 84 for the Reynolds number of 100 through 900 with the incremental of 100 , respectively. Under-relaxation is not used.

The computed results(streamlines, pressure contours, and vorticity contours) for $R e=$ 400,500 and 800 are shown in Figure 6,7 and 8. The computed pressure is adjusted by a constant such that $p=0$ at the lower step corner. The reattachment length of the recirculating zone behind the step and the location of detachment and reattachment of another recirculating zone near the upper wall are compared with experimental data in 
Figure 9, where $x_{1}$ is the reattachment location of the primary vortex, $x_{4}$ is the separation location of the secondary vortex at the top wall, $x_{5}$ is the reattachment location of the secondary vortex, and the distance is measured from the expansion step.

\section{CONCLUSIONS}

A new finite element method for incompressible Navier-Stokes problems is developed. This method is simple, robust and reliable. This method represents a particular application of a unified least-squares finite element method for first-order partial differential equations in computational physics. Further developments are under way for solving 3D problems and time-dependent problems. Theoretical investigation is also needed.

\section{REFERENCES}

1. P.S.Huyakorn, C.Taylor, R.L.Lee, and P.M.Gresho, A comparison of various mixedinterpolation finite elements in the velocity-pressure formulation of the Navier-Stokes equations, Computers and Fluids,6, 25-35(1978).

2. C.Taylor and T.G.Hughes, Finite Element Programming of the Navier-Stokes Equation, Pineridge Press, Swansea(1980).

3. G.F.Carey and J.T.Oden, Finite Elements: Fluid Mechanics, Vol.IV, Prentice-Hall(1986).

4. V.Girault and P.-A.Raviart, Finite Element Method for Navier-Stokes Equation, SpringerVerlag(1986).

5. C.Cuvelier, A.Segal and A.A.Van Steenhoven, Finite Element Methods and NavierStokes Equations, D.Reidel Publishing Company(1986).

6. C.Taylor(editor), Finite-Element Applications in Fluid Mechanics, in Finite Element Handbook,(editor-in-chief H.Kardestuncer), McGraw-Hill(1987).

7. I.Babuska, Error bounds for finite element method, Numer. Math.,16,322-333(1971).

8. F.Brezzi, On the existence, uniqueness and approximation of saddle-point problems arising from Lagrange multipliers, Rech. Oper., Ser. Rouge Anal. Numer.,8, R2,129-151(1974).

9. G.Comini and S.D.Giudice, Finite element solution of the compressible Navier-Stokes equations, Numer. Heat Transfer, 5, 463-478(1982).

10. A.C.Benim and W.Zinser, A segregated formulation of Navier-Stokes equations with finite elements, Comput. Meth. Appl. Mech. Engng., 57, 223-237(1986).

11. J.G.Rice and R.J.Schnipke, An equal-order veleocity-pressure formulation that does not exibit spurious pressure modes. Comput. Meth. Appl. Mech. Engng., 58, 135149(1986).

12. J.L.Sohn, Y.Kim and T.J.Chung, Finite element solver for incompressible fluid flows 
and heat transfer, in Finite Element Analysis in Fluids, (eds. T.J.Chung and G.R.Karr), Proceedings of the Seventh International Conference on Finite Element Methods in Flow Problems, UAH press, Huntsville, Alabama, 1989, pp.880-885.

13. B.N.Jiang and C.L.Chang, Least-squares finite elements for Stokes problem, Comput.Meth.Appl.Mech.Engrg.,(to appear); also available as NASA TM 101308, ICOMP88-16.

14. B.N.Jiang and L.A.Povinelli, Least-squares finite element method for fluid dynamics, in Finite Element Analysis in Fluids, (eds. T.J.Chung and G.R.Karr), Proceedings of the Seventh International Conference on Finite Element Methods in Flow Problems, UAH press, Huntsville, Alabama, 1989, pp.105-110.

15. B.N.Jiang and L.A.Povinelli, A least-squares finite element method for incompressible Navier-Stokes problem, in Proceedings of 12th Canadian Congress of Applied Mechanics,(eds. M.A.Erki and J.Kirkhope), Carleton University, Ottawa, Ontario, 1989, pp.602-603.

16. C.L.Chang and B.N.Jiang, An error analysis of least-squares finite element method of velocity-pressure-vorticity formulation for Stokes problem, Comput.Meth.Appl.Mech. Engng., (submitted).

17. B.N.Jiang and L.A.Povinelli, Least-squares finite element method for fluid dynamics, Comput. Meth. Appl. Mech. Engng., (to appear); also available as NASA TM 102352, ICOMP-89-23.

18. G.F.Carey and J.T.Oden, Finite Elements: A Second Course, ,Vol. II, Prentice-Hall, Englewood Cliffs, NJ,1983.

19. J.T.Oden and G.F.Carey, Finite Elements: Mathematical Aspects, Vol.IV, PrenticeHall, Englewood Cliffs, NJ, 1983.

20. G.F.Carey and B.N.Jiang, Element-by-element linear and nonlinear solution schemes, Communications in Applied Numerical Methods, 2, 145-153(1986).

21. G.F.Carey and E.J.Barragy, Parallel element-by-element solution scheme, Inter.J. $\mathrm{Nu}$ mer.Meth.Engng., 26, 2367-2382(1988).

22. A.K.Aziz, R.B.Kellogg and A.B.Stephens, Least-squares methods for elliptic systems, Mathematics of Computation,44, 53-70(1985).

23. G.F.Carey and B.N.Jiang, Least-squares finite element method and preconditioned conjugate gradient solution, Inter.J.Numer.Meth.Engng., 24, 1283-1296(1987).

24. E.D.Eason, A review of least-squares methods for solving partial differencial equations, Inter.J.Numer.Meth.Engng.,10, 1021-1046(1976).

25. U.Ghia, K.N.Ghia and C.T.Shin, High-Re solutions for incompressible flow using the Navier-Stokes equation and a multigrid method, J.Comput.Phys., 48, 387-411(1982).

26. P.M.Gresho, S.T.Chan, R.L.Lee and C.D.Upson, A modified finite element method for solving the time-dependent incompressible Navier-Stokes equations, Part 2. applications, Inter.J.Numer.Meth.Fluids, 4, 619-640(1984).

27. S.W.Kim, A fine grid finite element computation of two-dimensional high Reynolds number flows, Computers and Fluids, 16, 429-444(1988). 
28. B.F.Armaly, F.Durst, J.C.F.Pereira and B.Schonung, Experimental and theoretical investigation of backward-facing step flow, J.Fluid Mech.,127, 473-496(1982).



Figure 1. Finite element mesh $(50 \times 50$ bilinear elements $)$ for the cavity flow 


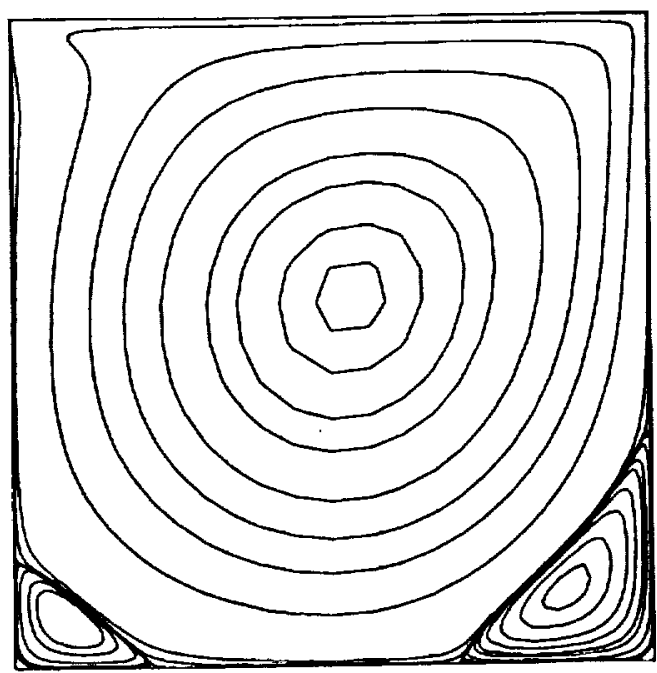

(a) Streamline

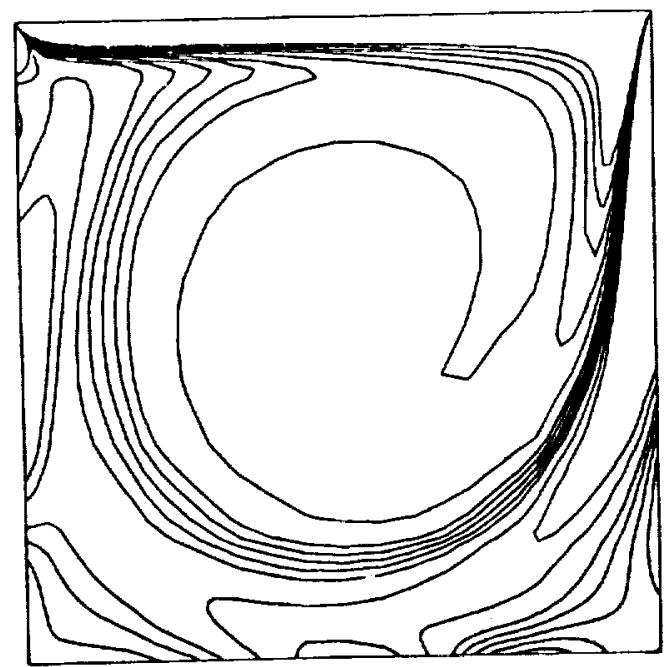

(c) Vorticity

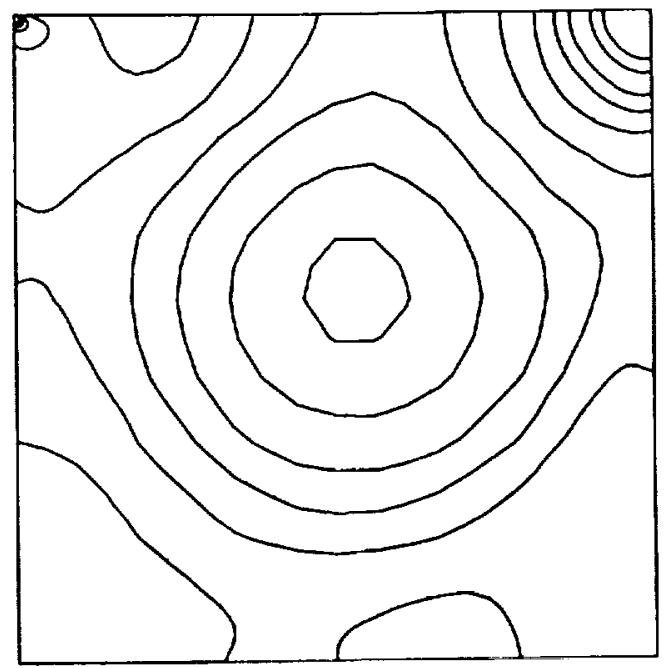

(b) Pressure



(d) Velocity

Figure 2. Computed results for cavity flow at $\operatorname{Re}=1000$ 


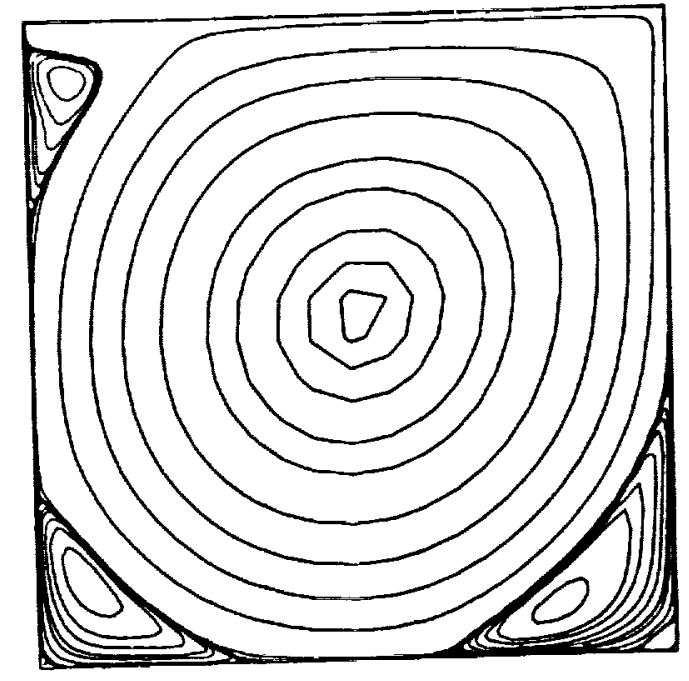

(a) Streamline

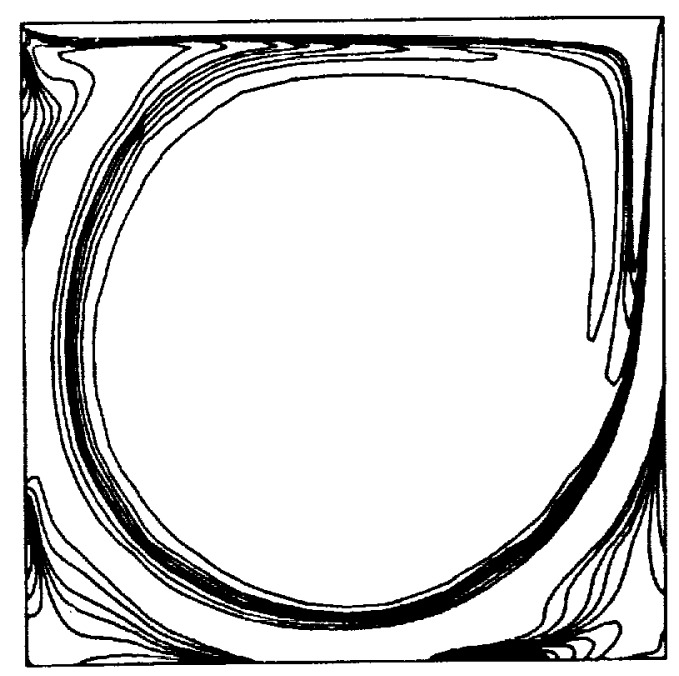

(c) Vorticity

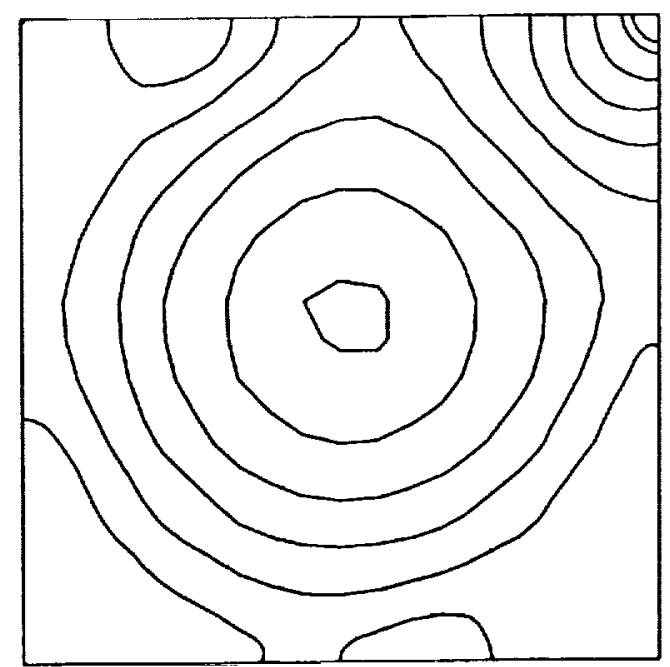

(b) Pressure

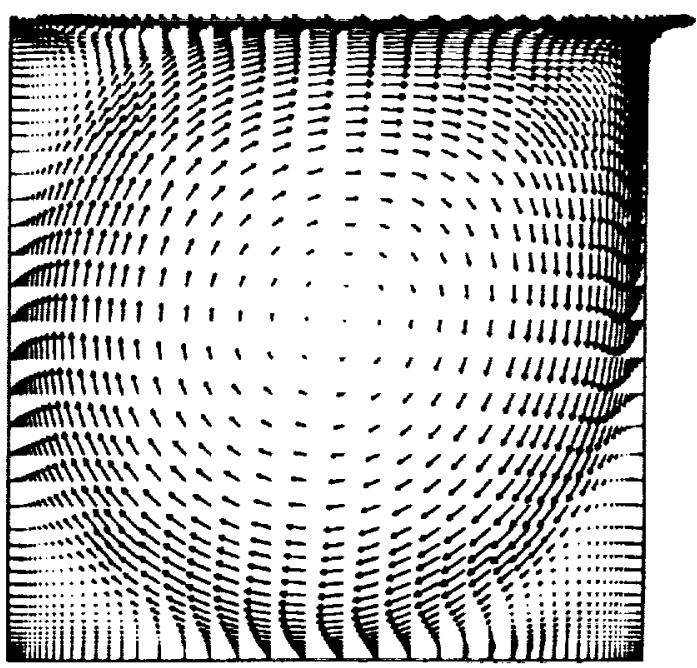

(d) Velocity

Figure 3. Computed results for cavity flow at $\mathrm{Re}=5000$ 


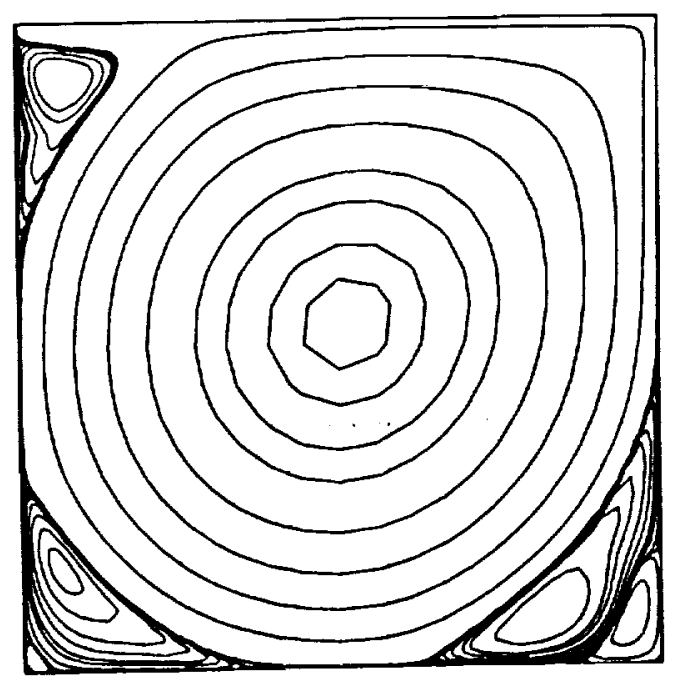

(a) Streamline

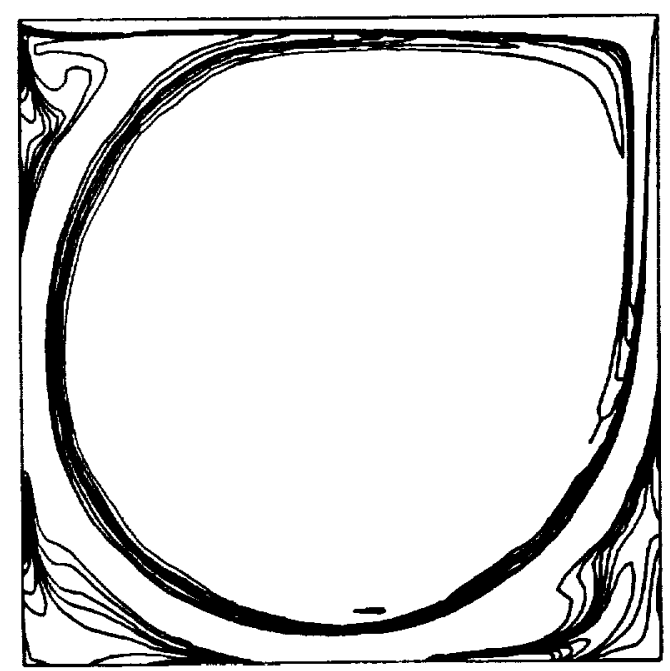

(c) Vorticity

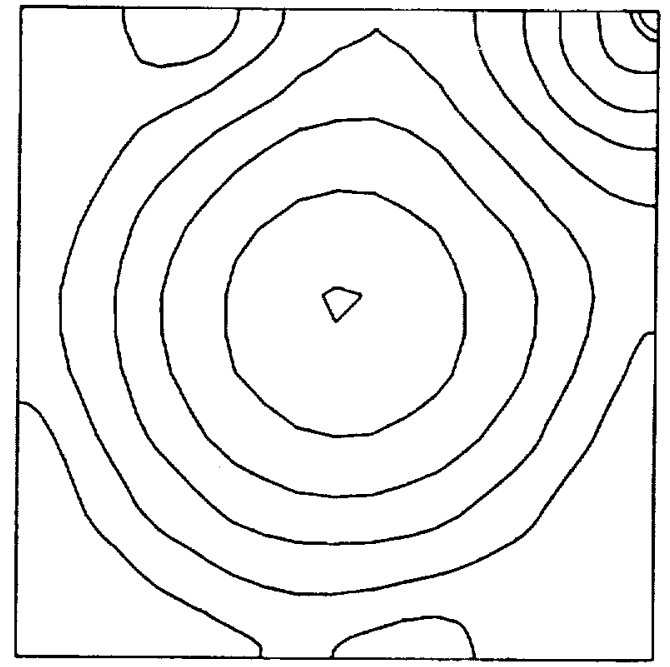

(b) Pressure



(d) Velocity

Figure 4. Computed results for cavity flow at $R e=10000$ 


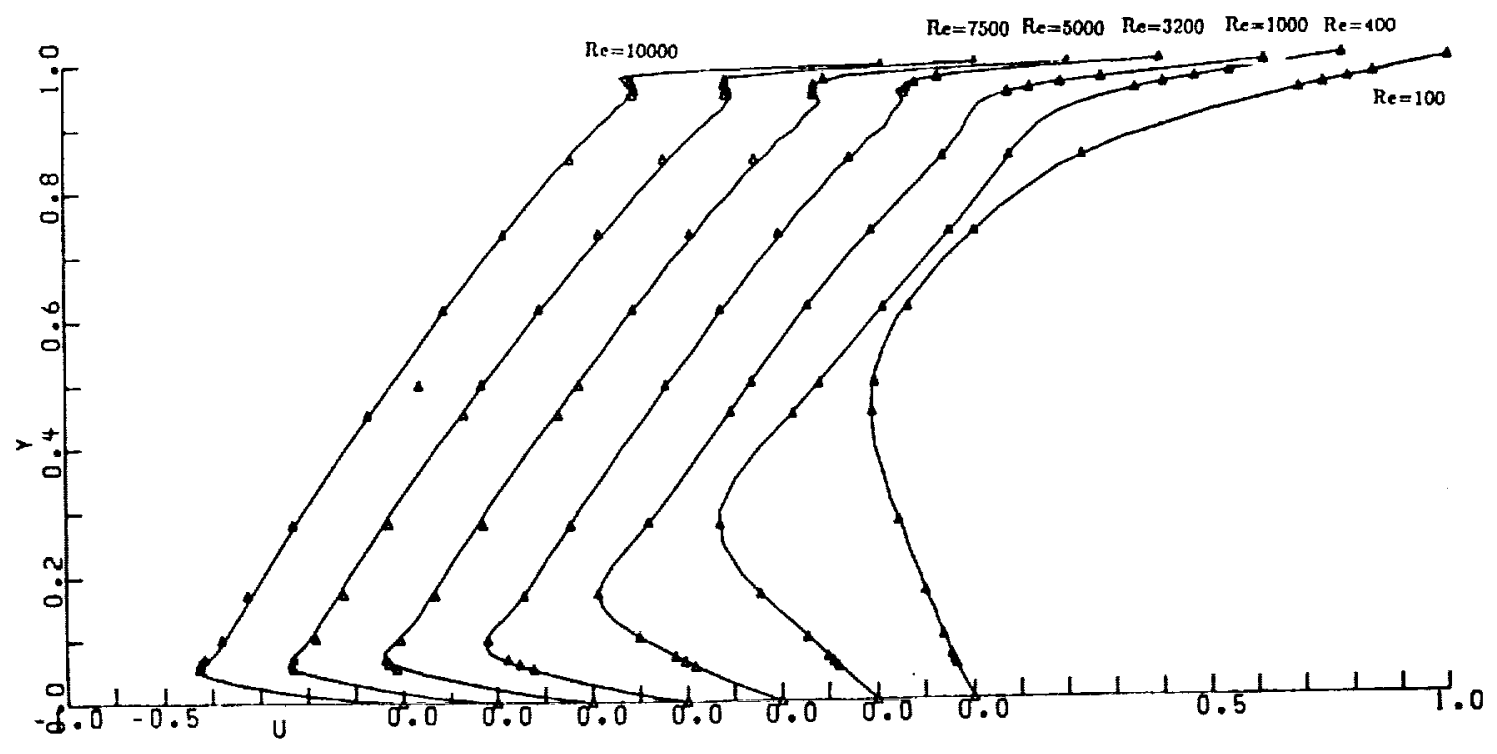

Figure 5. Horizontal velocity profile for cavity flow - present, $\triangle$ Ghia et a $l^{25}$ 


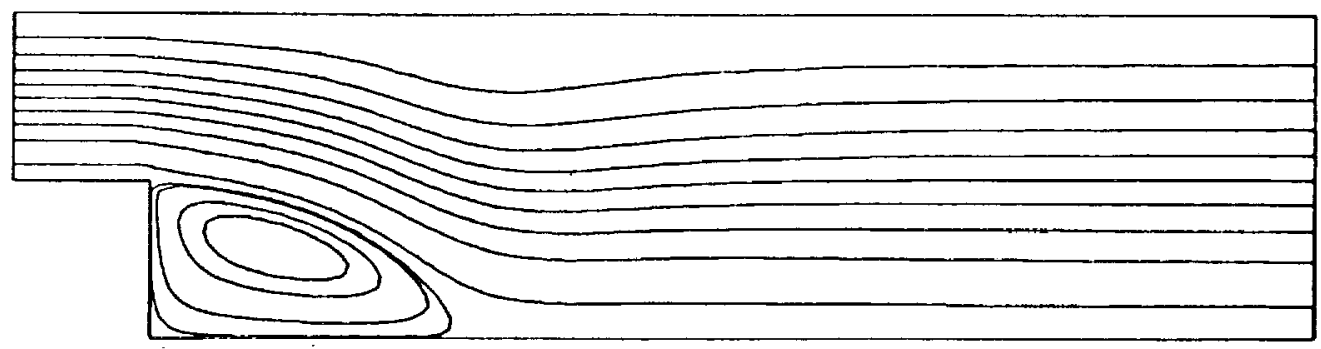

(a) Streamline



(b) Pressure

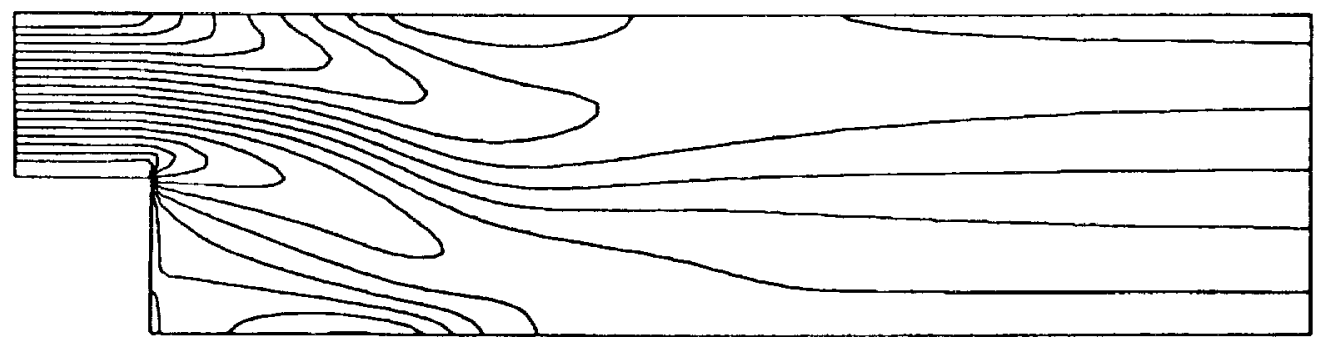

(c) Vorticity

Figure 6. Computed Results for Backward-Facing Step Flow at $\mathrm{Re}=400$. 


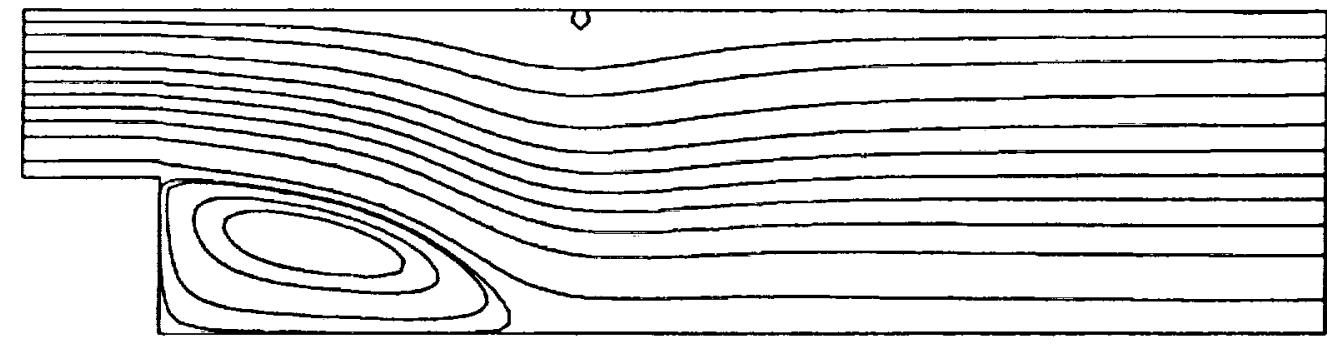

(a) Streamline

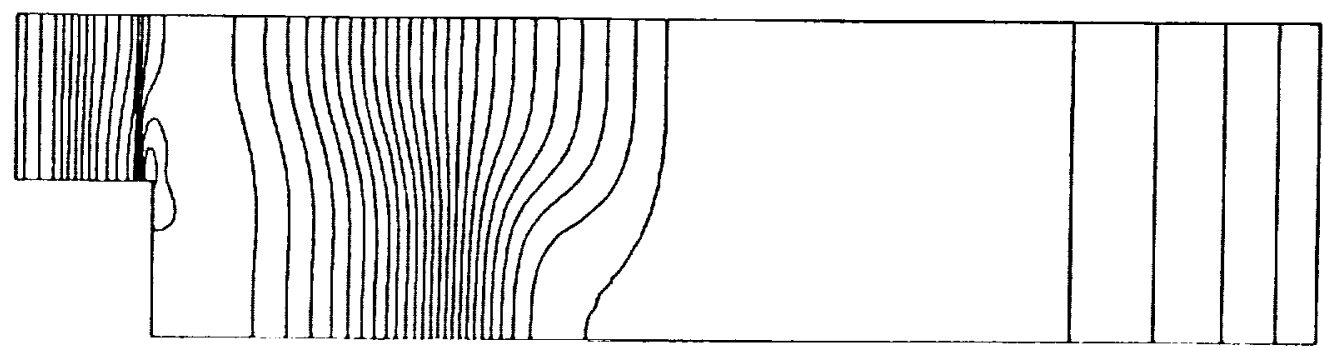

(b) Pressure

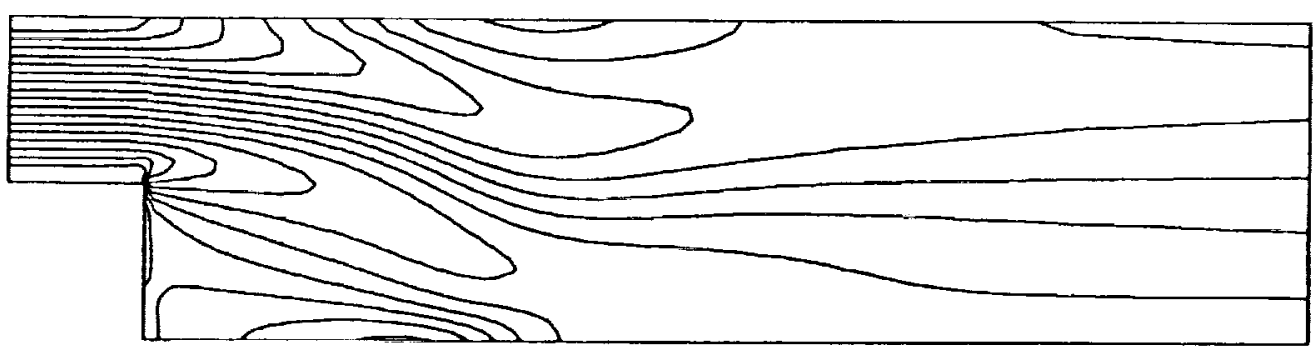

(c) Vorticity

Figure 7. Computed Results for backward-Facing Step Flow at $R e=500$. 


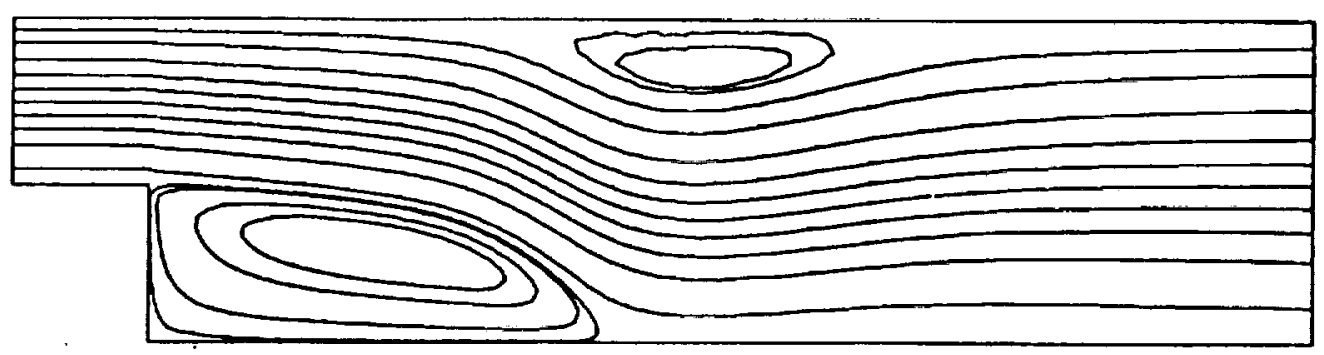

(a) Streamline

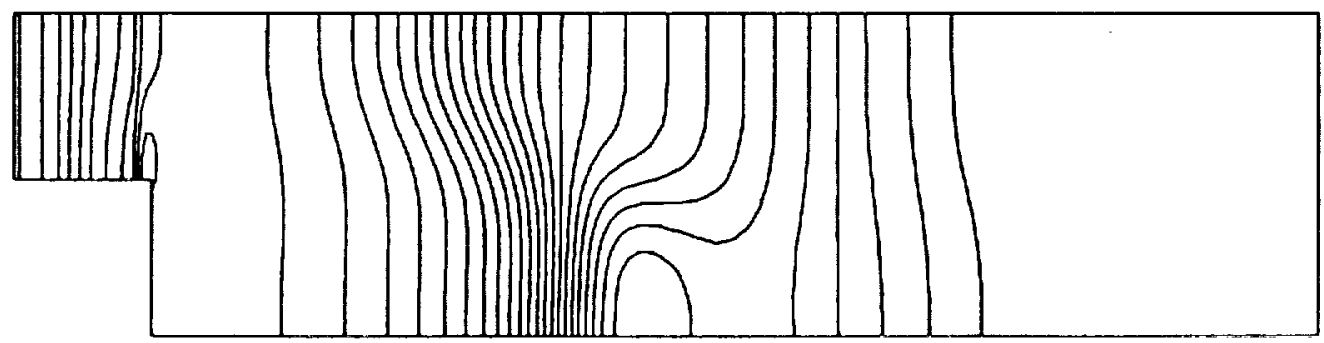

(b) Pressure



(c) Vorticity

Figure 8. Computed Results for Backward-Facing Step Flow at $\mathrm{Re}=800$ 


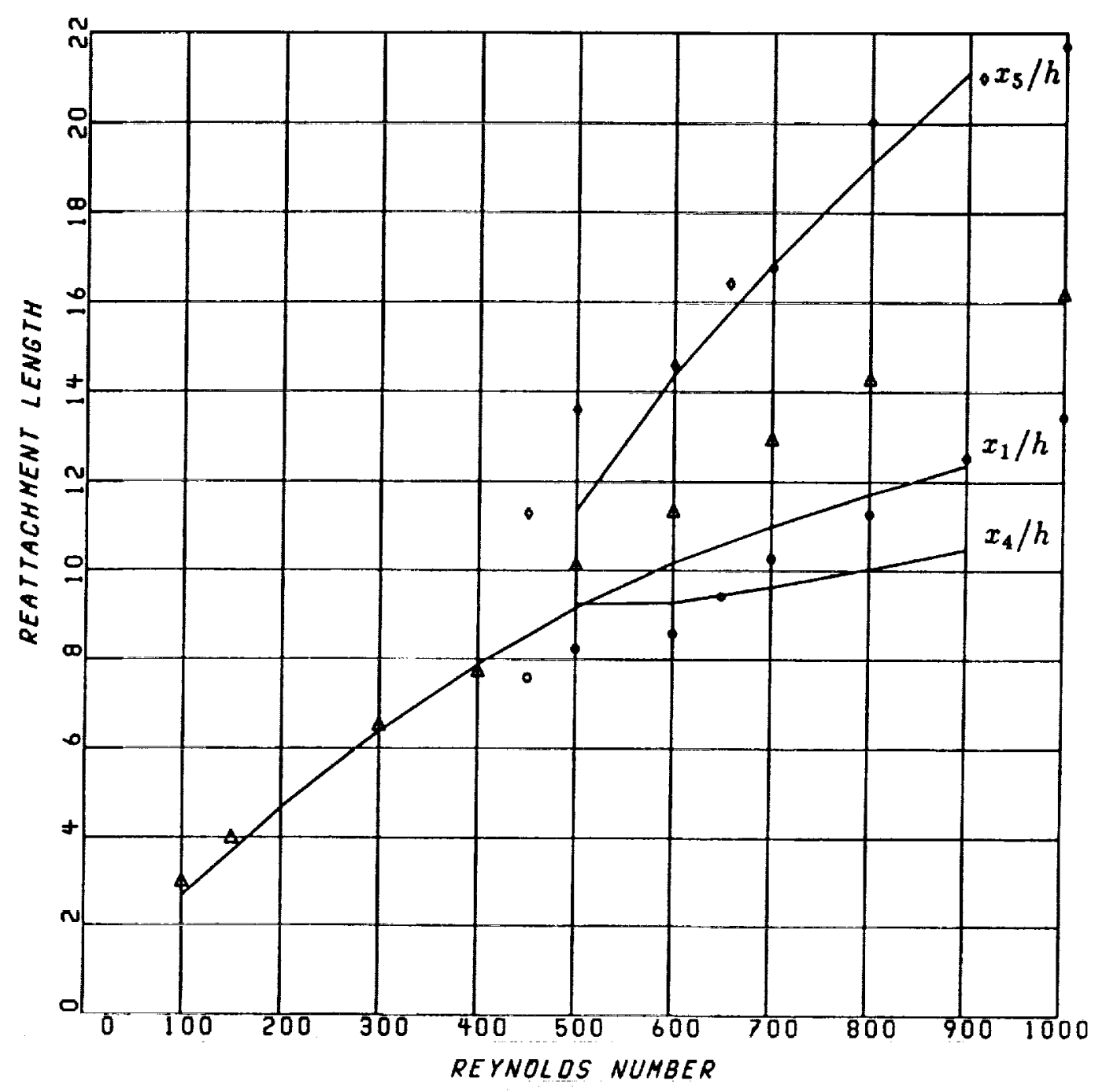

Figure 9. Reattachment length vs Reynolds number for backward-facing step flow - present, $\triangle, \circ, \diamond:$ Exp't $x_{1} / h, x_{4} / h, x_{5} / h$, respectively 




